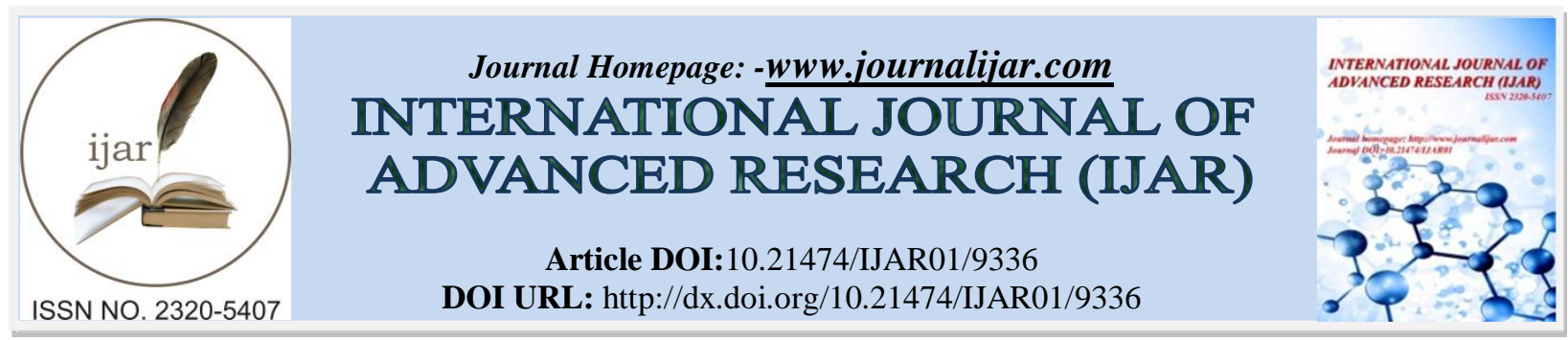

RESEARCH ARTICLE

\title{
LES DÉTERMINANTS DU CHANGEMENT DE L'AUDITEUR EXTERNE : CAS DES ENTREPRISES TUNISIENNES COTÉES DETERMINANTS OF AUDITOR SWITCH: EVIDENCE FROM TUNISIA.
}

Soumaya Ayedi.

\section{Manuscript Info}

Manuscript History

Received: 04 May 2019

Final Accepted: 06 June 2019

Published: July 2019

Key words:-

auditor switch, change in management leadership, long-term debt, qualified opinion.

\begin{abstract}
This study aims to identify the determinants of the auditor's switch within the Tunisian context. The results of the empirical analysis performed on a panel data and conducted on a sample of 23 nonfinancial listed Tunisian companies show that the change in the management leadership is a potential determinant of the auditor's switch. This result has also been confirmed within the frame of the robustness tests. We have also shown that the long-term debt fluctuation and the qualified opinion audit report are factors related to the decision of changing the auditor.
\end{abstract}

Copy Right, IJAR, 2019,. All rights reserved.

\section{Introduction:-}

La succession des scandales économiques a été à l'origine de la multiplication des recherches concernant la nécessité de mandater le changement d'auditeur et l'effet éventuel de cet amendement sur la qualité de l'audit (Corbella et al., 2015 ; Patterson et al., 2019). En effet, la majorité des études antérieures ont montré la nécessité d'un changement d'auditeur en vue de préserver l'indépendance du professionnel et ainsi de renforcer la qualité d'audit (Ball et al., 2015). Cependant, certains chercheurs ont témoigné qu'un changement d'auditeur peut être préjudiciable à l'entité auditée au même titre que l'auditeur en place (Cameran et al., 2016 ; Patterson et al., 2019) et qu'une longue durée de mandat contribue à l'amélioration de la qualité d'audit. Globalement, ces études ne sont pas intéressées à l'explication des facteurs déterminants la décision du changement de l'auditeur. Dans ce cadre, s'insère la problématique du présent travail cherchant à cerner les facteurs explicatifs du changement de l'auditeur externe dans le contexte tunisien. Nous désignons par un changement d'auditeur le fait que l'audité ne procède pas au renouvellement du mandat de l'auditeur en place, soit pour se conformer à la législation en vigueur, soit volontairement. Nous nous intéressons particulièrement à expliquer le changement de l'auditeur par des considérations contractuelles dans les entreprises tunisiennes cotées.

Le papier est organisé comme suit. La première partie synthétise les travaux antérieurs portant sur les déterminants du changement d'auditeur. Ensuite, nous allons développer nos hypothèses de recherche. La troisième partie synthétisera notre approche méthodologique. Les résultats de notre étude seront présentés dans le cadre de la quatrième partie. Une conclusion couronnera le présent papier.

\section{Revue de la littérature}

L'examen de la littérature antérieure a montré que le changement d'auditeur peut être à l'origine d'une application de la loi (Kuo et al., 2016) ou d'une décision prise par la société auditée. Différents facteurs peuvent expliquer la décision d'un changement volontaire de l'auditeur. Dans ce sens, un changement des caractéristiques du client 
audité peut être à l'origine d'une décision de changement de l'auditeur. Ainsi, Chadegani et al. (2011), Faiza et al. (2012) et Khasharmeh (2015) montrent que le changement du dirigeant est une cause potentielle du changement de l'auditeur. Par ailleurs, Schwartz et Menon (1985) montrent que les entreprises en situation de faillite ont plus tendance à changer d'auditeur que les entreprises saines. Les auteurs expliquent que la situation de détresse financière engendre un changement fréquent de l'équipe dirigeante, l'émission de rapports d'audit assortis de réserves et par conséquent contribue à la décision d'un changement d'auditeur. Johnson et Lys (1990) montrent l'existence d'un impact significatif de l'endettement sur la décision d'un changement d'auditeur. DeFond (1992) montre que les dirigeants s'engagent à changer l'auditeur, lorsque les coûts d'agence s'intensifient ou lorsqu'ils sont capables d'anticiper une telle intensification. Huson et al. (2000) montrent que le changement de l'auditeur est influencé par un accroissement important du chiffre d'affaire, du total actif, de l'endettement et partiellement par un changement du dirigeant.

Un changement des caractéristiques de l'auditeur peut expliquer aussi le changement de l'auditeur. Ainsi, Choy et al. (2019) montrent que dans le contexte taïwanais, les entreprises cotées ont tendance à changer leur auditeur dès lors que celui-ci élargit son portefeuille et par conséquent n'a plus la capacité de répondre aux besoins de toute sa clientèle. Chang et al. (2019) montrent que les clients taïwanais ont tendance à changer d'auditeur et suivre le partenaire d'audit avec lequel ils travaillaient pendant longtemps lorsque celui-ci quitte le cabinet d'audit auquel il appartenait, ceci étant pour les clients des 'Non Big4'. En plus, Francis et al. (2017) montrent que les auditeurs qui perdent la majorité de leur clientèle appartenant à un même secteur d'activité perdent leur réputation. Ceci aura pour effet de perdre le restant des clients appartenant au même secteur d'activité. Par ailleurs, l'environnement d'audit peut être aussi à l'origine de la décision du changement de l'auditeur. Ainsi, Rennie et al. (2014) montrent que les audités peuvent devenir intimidants à l'égard de leurs auditeurs, en exerçant sur eux une pression énorme en vue de rediriger leurs domaines d'intervention ou leurs conclusions. Un mécontentement de la part de l'audité vis-à-vis de son auditeur peut être à l'origine de son changement. Heliodoro et al. (2016) et Darmayanti (2017) montrent aussi que les entreprises ayant reçu un rapport d'audit assorti de réserves ont tendance à changer leur auditeur.

Dans le contexte tunisien, un des pays émergents, l'auditeur est généralement désigné par l'assemblée générale des actionnaires pour une durée irrévocable de trois années consécutives. Le changement de l'auditeur est mandaté à l'expiration du troisième mandat successif ${ }^{1}$. Cependant, un changement volontaire peut avoir lieu avant l'expiration de la durée légale et relèvera dans ce cas de la propre décision de l'assemblée générale. Cette recherche s'inscrit dans ce cadre et elle cherche à expliquer le changement d'auditeur chez les entreprises tunisiennes essentiellement par des considérations contractuelles. La partie suivante sera réservée au développement des hypothèses de recherche.

\section{Hypothèses de recherche}

La présente étude vise à déterminer les facteurs pouvant expliquer le changement d'auditeur chez les entreprises tunisiennes cotées. Elle se base essentiellement sur les prédictions de la théorie d'agence.

\section{Propriété managériale et changement d'auditeur}

Jensen et Meckling (1976) perçoivent l'entreprise comme un nœud de contrats conclus entre différents agents économiques rationnels, le dirigeant est au centre du nœud. Dans la mesure où il ne détient pas une part importante du capital de l'entreprise, le dirigeant devrait gérer les fonds mis à sa disposition conformément à ses propres intérêts. L'audit est ainsi préconisé comme étant une solution aux conflits d'agence entre les actionnaires et les dirigeants. Le besoin d'une meilleure qualité d'audit est généralement expliqué par l'intensité des coûts découlant des relations d'agence entre les actionnaires et les dirigeants. L'examen de la littérature antérieure révèle l'hétérogénéité des résultats concernant le pouvoir explicatif des coûts d'agence sur la décision du changement d'auditeur et aussi de la direction de ce changement. En effet, plusieurs études montrent qu'un accroissement des coûts d'agence engendre un changement d'auditeur et la demande d'une meilleure qualité d'audit (DeFond, 1992). Toutefois, Chen et Zhou (2007) montrent que les variables d'agence, notamment celles actionnaires-dirigeants n'ont pas d'effet sur la décision du changement de l'auditeur externe. Le contexte tunisien est caractérisé par une prépondérance des entreprises familiales dirigées généralement par l'un des membres de la famille. Ceci devrait atténuer les conflits d'agence actionnaires-dirigeants. Toutefois, dans la mesure où le dirigeant ne détient pas une

${ }^{1}$ La loi 2005-96 relative à la sécurité des relations financières a prévu une limitation au renouvellement du mandat de l'auditeur des sociétés soumises à l'obligation de désigner un commissaire aux comptes inscrit au tableau de l'Ordre des Experts Comptables de la Tunisie. 
part assez importante du capital de l'entreprise qu'il gère, il sera amené à privilégier ses propres intérêts. Ceci renforce le besoin des actionnaires en contrôle des actions du dirigeant qui se matérialise par une décision de changement d'auditeur. La première hypothèse est ainsi formulée comme suit.

\section{Hypothèse 1 :}

La propriété managériale influence la décision du changement de l'auditeur externe.

\section{Concentration de propriété et changement d'auditeur}

L'entreprise est le lieu de rencontre potentiel des actionnaires majoritaires et des actionnaires minoritaires. Les actionnaires majoritaires sont les actionnaires influents au sein des entreprises, à qui revient la prise des décisions stratégiques. Selon la théorie d'agence, les actionnaires majoritaires entreprennent des décisions qui tendent à exproprier la richesse des actionnaires minoritaires. D'où la naissance de conflits d'agence actionnaires majoritairesactionnaires minoritaires. L'audit est l'un des moyens servant à protéger les intérêts des actionnaires minoritaires. Le problème qui persiste là est lorsque le capital des entreprises devient de plus en plus concentré, le risque d'expropriation de la richesse des actionnaires minoritaires par les détenteurs de blocs augmente. Dans ce sens, Aroh et al. (2017) montrent qu'au Nigeria, la concentration de propriété constitue un facteur déterminant de la décision de changement d'auditeur. Dans le contexte tunisien, la décision de désignation et de changement de l'auditeur est prise par les actionnaires représentant la majorité des voix dans l'assemblée générale. Dans ce sens, nous considérons l'impact éventuel de la concentration de propriété sur le changement de l'auditeur.

\section{Hypothèse 2 :}

La concentration du capital influence la décision du changement de l'auditeur externe.

\section{Endettement et changement d'auditeur}

Conformément aux avancées de la théorie d'agence, les créanciers mettent à la disposition des actionnaires des fonds en contrepartie d'un remboursement et d'une rémunération afférente à une échéance préalablement fixée. Les actionnaires étant des agents opportunistes cherchent à détourner les fonds mis à leur disposition au détriment des intérêts des créanciers. Un conflit d'intérêt entre les actionnaires et les créanciers est alors né. L'auditeur intervient dans ce cadre en vue de certifier les états financiers de l'entreprise et de rassurer les créanciers sur la solvabilité et la pérennité de l'entreprise à qui ils ont confié leurs fonds. En quête de nouvelles sources de financement externes, les actionnaires chercheront à accroitre la fiabilité des états financiers à travers le changement de l'auditeur en place. Ceci a été documenté dans la littérature antérieure (DeFond, 1992 ; Huson et al., 2000 ; Shahnaz et al., 2008 ; Faiza et al., 2012) prouvant que l'endettement est associé au changement de l'auditeur externe. Le contexte tunisien est caractérisé par un faible dynamisme du marché financier et le recours prépondérant des entreprises tunisiennes à l'endettement, surtout à long terme, comme moyen privilégié de financement. Nous nous attendons ainsi à ce que l'endettement soit un facteur déterminant la décision de changement de l'auditeur.

\section{Hypothèse 3 :}

L'endettement influence la décision du changement de l'auditeur externe.

\section{Insertion de réserves dans le rapport d'audit et changement d'auditeur}

Darmayanti (2017) montre que l'insertion de réserves dans le rapport d'audit constitue une source de conflit entre l'auditeur et l'audité, qui peut mener à un changement d'auditeur. En effet, l'audité engage l'auditeur pour donner un avis sur la sincérité des comptes qu'il présente annuellement. Toutefois, l'audité veut se prémunir contre la réception de toute réserve pour ne pas être pénalisé sur le marché financier et avoir des obstacles face à l'obtention de nouvelles sources de financement externe. L'auditeur ne désirant pas engager sa responsabilité et compromettre son indépendance vis-à-vis de son client, il aura tendance à révéler toute erreur ou fraude découverte à l'occasion de son intervention. L'audité tend ainsi à changer vers un auditeur de plus faible qualité d'audit (Aguilar et Barbadillo, 2003) afin de se prémunir de l'insertion des réserves dans le rapport d'audit. La loi tunisienne sanctionne tout auditeur n'ayant pas révélé dans son rapport d'audit un fait délictueux ou une erreur découverte dans les états financiers contrôlés. L'auditeur est ainsi appelé à procéder à l'émission de rapport d'audit assorti de réserves en cas de besoin.

\section{Hypothèse 4 :}

L'insertion de réserves dans le rapport d'audit influence la décision du changement de l'auditeur externe. 


\section{Changement du dirigeant et changement d'auditeur}

En raison d'une mauvaise gestion ou d'un conflit sur les principes de gestion de l'entreprise, les actionnaires cherchent dans la plupart des cas à remplacer le dirigeant en place. Le nouveau dirigeant recruté aura pour objectif l'amélioration de la situation financière de l'entreprise et le renforcement de la confiance des investisseurs en la prospérité de l'entreprise qu'il gère. Il cherchera ainsi à changer d'auditeur avec qui il a une relation personnelle (Beattie et Fearnley, 1998) ou un auditeur fortement réputé. La littérature antérieure montre que le changement du dirigeant peut être à l'origine d'un changement d'auditeur (Hudaib et Cooke, 2005; Chadegani et al., 2011 ; Khashermeh, 2015). Dans le contexte tunisien, l'assemblée générale des actionnaires reste le principal acteur décidant le changement d'auditeur et la désignation du nouveau professionnel. Toutefois, étant donné que les relations personnelles priment dans l'environnement économique tunisien, le dirigeant peut influencer ou diriger les décisions prises en assemblée des actionnaires. En effet, Piot (2003) montre que le dirigeant intervient directement ou indirectement dans le processus de désignation des auditeurs dans le cadre des entreprises françaises cotées.

\section{Hypothèse 5 :}

Le changement du dirigeant influence la décision du changement de l'auditeur externe.

Nous décrivons dans la suite notre approche méthodologique.

\section{Approche méthodologique:-}

Nous identifions notre échantillon, notre modèle d'étude ainsi que la méthode d'analyse adoptée.

\section{Echantillon}

Notre échantillon d'étude correspond à 23 entreprises tunisiennes cotées sur le marché principal de la BVMT et la période d'étude s'étale entre 2007 et 2017, soit 253 observations. Toutes les données nécessaires ont été collectées à partir de la consultation des états financiers définitifs des entreprises cotées et de leurs rapports d'audit disponibles sur le site web de la BVMT.

\section{Modèle d'étude}

1. Rappelons que notre objectif est d'identifier les déterminants du changement d'auditeur dans le contexte tunisien. Il s'agit de considérer une mesure binaire de la variable dépendante changement d'auditeur (Shahnaz et al., 2008 ; Tu, 2012 ; Zhang et al., 2019) tel que CHAUD $_{\text {it }}=1$ si l'entreprise ${ }_{\mathrm{i}}$ a procédé au changement de son auditeur à la fin de l'année $t, 0$ sinon.

2. Les variables indépendantes introduites dans le modèle sont les suivantes :

3. La propriété managériale (PRMANG) est mesurée par le pourcentage d'actions détenues par le dirigeant de l'entreprise.

4. La concentration de la propriété (PLACT) est mesurée par le pourcentage du capital détenu par les trois principaux actionnaires.

5. L'endettement (LTDTA) est mesuré par le ratio d'endettement à terme de l'entreprise.

6. Le changement du dirigeant (CHDRG), s'agissant d'une variable dichotomique prenant la valeur 1 si l'entreprise a procédé à un changement de son PDG ou DG et 0 sinon.

7. L'insertion de réserves dans le rapport d'audit (RESV) prenant la valeur 1 si le rapport d'audit de l'entreprise est assorti de réserves et 0 sinon.

8. Nous contrôlons l'effet de la variable taille de l'entreprise, tel que LNACTF est égale au logarithme naturel du total actif de l'entreprise.

Le modèle d'étude se présente alors ainsi :

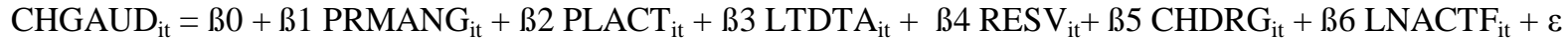

Le tableau 1 qui suit récapitule l'ensemble des variables de l'étude.

Tableau 1:-Récapitulatif des variables de l'étude

\begin{tabular}{|l|l|l|l|}
\hline & \multicolumn{1}{|c|}{ Désignation } & \multicolumn{1}{c|}{ Mesure } & Littérature antérieure \\
\hline $\begin{array}{l}\text { Variable } \\
\text { dépendante }\end{array}$ & $\begin{array}{l}\text { CHAUD : } \\
\text { Changement } \\
\text { d'auditeur }\end{array}$ & $\begin{array}{l}\text { 1 si l'entreprise a pas procédé au } \\
\text { changement de son auditeur, 0 sinon. }\end{array}$ & $\begin{array}{l}\text { Shahnaz et al. (2008), } \\
\text { Zhang et al. (2019) }\end{array}$ \\
\hline Variables d'intérêt & PRMANG : Propriété & Pourcentage de capital détenu par le & DeFond (1992), Chen et \\
\hline
\end{tabular}




\begin{tabular}{|c|c|c|c|}
\hline & managériale & dirigeant. & Zhou (2007) \\
\hline & $\begin{array}{l}\text { PLACT : } \\
\text { Concentration } \\
\text { propriété }\end{array}$ & $\begin{array}{l}\text { Pourcentage d'actions détenues par } \\
\text { les trois principaux actionnaires }\end{array}$ & Aroh et al. (2017) \\
\hline & $\begin{array}{l}\text { LTDTA : } \\
\text { Endettement total }\end{array}$ & $\begin{array}{l}\text { Dettes à long terme rapportées au } \\
\text { total actif. }\end{array}$ & Piot (2003) \\
\hline & $\begin{array}{l}\text { RESV : } \\
\text { Insertion de réserves } \\
\text { dans le rapport d'audit }\end{array}$ & $\begin{array}{l}\text { Variable binaire prenant la valeur } 1 \text { si } \\
\text { le rapport d'audit est assorti de } \\
\text { réserves, } 0 \text { sinon. }\end{array}$ & $\begin{array}{l}\text { Heliodoro et al. (2016), } \\
\text { Darmayanti (2017) }\end{array}$ \\
\hline & $\begin{array}{l}\text { CHDRG : } \\
\text { Changement du } \\
\text { dirigeant }\end{array}$ & $\begin{array}{l}\text { Variable binaire prenant la valeur } 1 \text { si } \\
\text { l'entreprise a changé son dirigeant, } 0 \\
\text { sinon. }\end{array}$ & $\begin{array}{l}\text { Tu (2012), Khasharmeh } \\
\text { (2015) }\end{array}$ \\
\hline $\begin{array}{l}\text { Variable de } \\
\text { contrôle }\end{array}$ & $\begin{array}{l}\text { LNACTF : Taille de } \\
\text { l'audité }\end{array}$ & $\begin{array}{l}\text { Logarithme népérien du total de } \\
\text { l'actif. }\end{array}$ & $\begin{array}{l}\text { Ghosh et Lustgarten } \\
(2006)\end{array}$ \\
\hline
\end{tabular}

\section{Méthode d'analyse}

Notre modèle d'étude sera testé empiriquement sur des données de panel. Il s'agit de recourir à la méthode logit sous le logiciel STATA. Ceci est justifié par le fait que la variable dépendante est du type dichotomique et que nous ne pouvons pas confirmer la normalité de l'ensemble des variables introduites dans le modèle.

\section{Résultats et discussion:-}

Nous présentons d'abord les statistiques descriptives des variables de l'étude (tableau 2). Ensuite nous procéderons à la vérification de l'absence d'un problème de multi-colinéarité entre les variables indépendantes introduites dans le modèle d'étude (tableau 3) et finir avec les résultats de issus de l'analyse multivariée (tableau 4).

\section{Résultats des statistiques descriptives}

Le tableau 2 suivant récapitule les moyennes, médianes, écart type et la valeur minimale et maximale des variables métriques et la fréquence par modalité pour les variables binaires.

Tableau 2:-Statistiques descriptives des variables de l'étude

\begin{tabular}{|c|c|c|c|c|c|}
\hline $\begin{array}{l}\text { Variables } \\
\text { métriques }\end{array}$ & Moyenne & Médiane & Ecart type & Minimum & Maximum \\
\hline PRMANG & 6.2 & 0 & 16.61 & 0 & 75.5 \\
\hline PLACT & 62.75 & 66 & 17.55 & 29 & 99.75 \\
\hline LTDTA & 0.11 & 0.08 & 0.12 & 0 & 0.71 \\
\hline LNACTF & 18.11 & 17.95 & 0.95 & 16.56 & 21.43 \\
\hline $\begin{array}{l}\text { Variables } \\
\text { binaires }\end{array}$ & \multicolumn{3}{|c|}{ Fréquence de la modalité 0} & \multicolumn{2}{|c|}{ Fréquence de la modalité 1} \\
\hline CHDRG & \multicolumn{3}{|c|}{245} & \multicolumn{2}{|c|}{8} \\
\hline RESV & \multicolumn{3}{|c|}{240} & \multicolumn{2}{|c|}{13} \\
\hline CHAUD & \multicolumn{3}{|c|}{210} & \multicolumn{2}{|c|}{43} \\
\hline
\end{tabular}

CHAUD $_{\text {it }}=1$ si l'entreprise i à l'année $(\mathrm{t})$ a procédé au changement de son auditeur de l'année (t-1), 0 sinon. PRMANG $_{i t}=$ Pourcentage d'actions détenues par le dirigeant de l'entreprise $_{(\mathrm{i})}$ à l'année $(\mathrm{t}) \cdot$ LTDTA $_{\mathrm{it}}=$ Dettes a long terme $_{\text {it }} /$ actif total $_{\text {it }} \cdot \mathrm{RESV}_{\mathrm{it}}=1$ si le rapport d'audit de l'entreprise i relatif à l'année t est assorti de réserves, 0 sinon. $\mathrm{CHDRG}_{\mathrm{it}}=1$ si un changement de dirigeant a été effectué au cours de l'exercice t, 0 sinon. $\mathrm{LNACTF}_{\mathrm{it}}=$ logarithme népérien du total actif.

Il est à remarquer que le capital des entreprises tunisiennes cotées est fortement concentré comme le démontre la valeur moyenne (et médiane) de la variable PLACT, avec une proportion de propriété managériale moyennement assez réduite (environ $6 \%$ ). L'endettement à terme est très hétérogène dans l'échantillon d'étude variant entre l'absence de dettes à une proportion avoisinant les $70 \%$. Les statistiques descriptives relatives aux variables CHDRG et CHAUD montrent que les entreprises tunisiennes cotées ne procèdent pas à un changement fréquent de 
leur dirigeant et de leur auditeur, respectivement. En effet, nous notons environ $3 \%$ de cas de changement de dirigeant sur toute la période de l'étude et environ $17 \%$ de cas de changement d'auditeur.

\section{Matrice de correlation:-}

Nous présentons dans le tableau 3 qui suit les coefficients de corrélation de Spearman entre toutes les variables explicatives de l'étude. Notre recours à ce test non paramétrique est expliqué par le fait que nous ne pouvons pas vérifier la linéarité toutes les variables explicatives.

Tableau 3:-Matrice de corrélation entre les variables indépendantes

\begin{tabular}{|c|c|c|c|c|c|c|}
\hline & PRMANG & PLACT & LTDTA & RESV & CHDRG & LNACTF \\
\hline PLACT & -0.0296 & & & & & \\
\hline LTDTA & -0.0941 & -0.0511 & & & & \\
\hline RESV & -0.0504 & 0.2072 & 0.2714 & & & \\
\hline CHDRG & 0.0166 & 0.0936 & 0.0532 & 0.1626 & & \\
\hline LNACTF & -0.2999 & 0.1104 & 0.3494 & 0.3325 & 0.1367 & 1.000 \\
\hline
\end{tabular}

Les coefficients de corrélation de Spearman figurant au tableau 3 précédent montrent qu'il n'existe aucun problème sérieux de multicolinéarité entre les variables indépendantes introduites dans le modèle de l'étude (Kervin, 1992).

\section{Résultats de l'estimation logit du modèle d'étude}

Le tableau 4 qui suit synthétise les résultats de l'estimation logit du modèle d'étude.

Tableau 4:-Estimation Logit du modèle d'étude

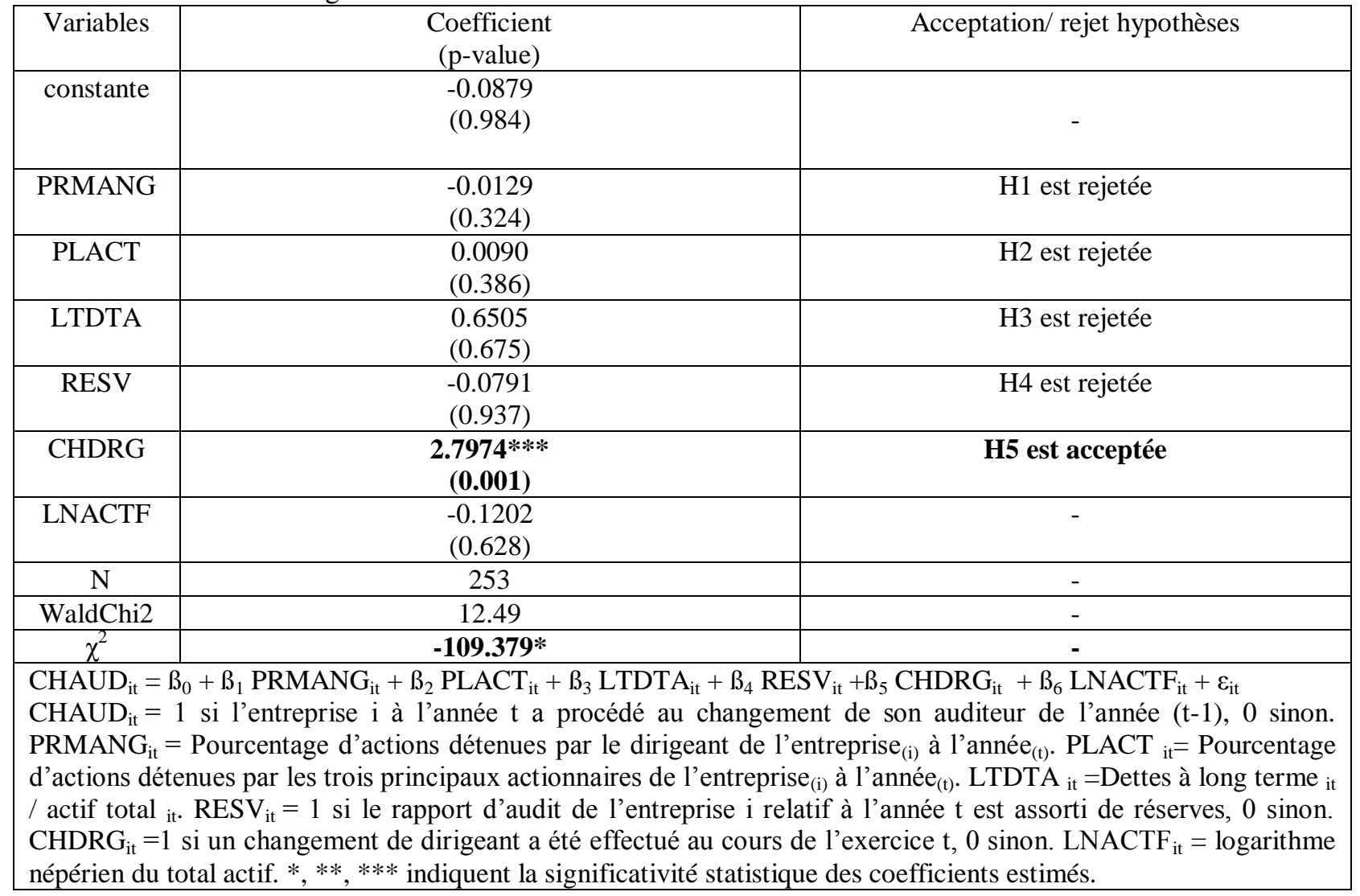

Les résultats figurant au tableau 4 montrent que la propriété managériale n'affecte pas la décision du changement de l'auditeur. D'où l'hypothèse 1 est rejetée. Ceci peut s'expliquer par le fait que dans les entreprises familiales où le dirigeant ne détient pas une part importante du capital, il n'exerce aucune influence sur les décisions de l'assemblée générale. De même, la concentration de propriété n'a pas d'influence sur la décision du changement de l'auditeur 
(Aroh et al., 2017) rejetant ainsi notre deuxième hypothèse. Ce résultat nous semble très intéressant à interpréter dans le cadre des entreprises tunisiennes étant donné que la décision de changement de l'auditeur revient en priorité aux actionnaires majoritaires. Nous pouvons expliquer ceci par la réticence des entreprises tunisiennes à changer leur auditeur fréquemment et par le poids des relations personnelles régnant dans le domaine professionnel. Par ailleurs, l'endettement à terme ne semble pas influencer la décision du changement de l'auditeur. Nous rejetons ainsi notre troisième hypothèse de recherche (Liu, 2007). Il paraît que dans le contexte tunisien, l'endettement seul ne permet pas de créer un environnement risqué incitant les actionnaires à changer d'auditeur. Il fallait notamment associer l'endettement à la présence de fortes opportunités d'investissement dans les entreprises relevant la barre de risque de non solvabilité et de non recouvrement des dettes à un très haut niveau (Piot, 2003). Concernant la variable insertion des réserves dans le rapport d'audit (RESV), les résultats de l'estimation Logit en données de panel du modèle d'étude montrent qu'elle n'exerce aucune influence significative sur le changement de l'auditeur, d'où le rejet de la quatrième hypothèse. Nous pouvons expliquer ces résultats par le fait que l'auditeur est protégé pendant trois années correspondant à la durée du mandat et durant lesquelles aucun changement ne peut être effectué même en cas d'insertion de réserves dans le rapport d'audit. Par ailleurs, il s'avère que le changement du dirigeant est un facteur déterminant le changement de l'auditeur dans le contexte tunisien. D'où l'acceptation de notre cinquième hypothèse. Ce résultat est en concordance avec les résultats de Piot (2003) dans le contexte français et de Darmayanti (2017) dans le contexte indonésien. Il semble qu'au sein des entreprises tunisiennes cotées le recrutement d'un nouveau dirigeant engendre un changement d'auditeur, et ce malgré que la décision de la désignation du CAC revient à l'assemblée générale de l'entreprise en question. Nous pouvons ainsi soutenir l'explication de la relation entre le changement du dirigeant et le changement d'auditeur fournie par Beattie et Fearnley (1998) tel qu'un nouveau dirigeant recruté cherche à changer vers un auditeur avec qui il a une relation personnelle ou un auditeur fortement réputé. Concernant la variable de contrôle introduite, à savoir la taille de l'entreprise (LNACTF), il paraît qu'elle n'exerce aucune influence sur la décision du changement d'auditeur.

Prenant en considération la protection du mandat de l'auditeur durant trois ans par le législateur, il serait intéressant de prévoir des tests de robustesse afin d'analyser l'effet de la variation dynamique sur trois ans des variables explicatives sur le changement de l'auditeur.

\section{Tests de robustesse}

Pour procéder à une analyse dynamique de l'impact des variables de l'étude sur le changement de l'auditeur, nous avons identifié sur la base de notre échantillon d'étude relatif à la période 2007-2017 les cas de renouvellement du mandat de l'auditeur externe et les cas de changement opérés. Pour se faire, nous nous sommes fiés à la date du début et de la fin du mandat de l'auditeur figurant sur les guides des sociétés cotées et/ou sur le rapport d'audit. Nous avons ainsi identifié 40 cas de renouvellement du mandat de l'auditeur et 43 cas de changement de l'auditeur. La nouvelle mesure adoptée à la variable dépendante CHAUD est la suivante :

CHAUD = 1 si l'entreprise a procédé au changement de son auditeur à la fin de son mandat, 0 sinon.

Afin de calculer les variations des variables explicatives de l'étude sur les trois années précédant le changement ou le renouvellement du mandat de l'auditeur, nous étions obligé de réduire notre échantillon de changement et de renouvellement du mandat de l'auditeur. L'échantillon est ainsi porté à 37 cas de changement d'auditeur et à 32 cas de renouvellement.Nous avons recouru à des tests non paramétriques de Mann-Whitney (ou du khi-deux pour les variables modales) pour échantillons indépendants, pratiqués sur la variation des variables étudiées sur la durée du mandat de l'auditeur.

Nous présentons dans ce qui suit les résultats des tests pour échantillons indépendants partagés selon les modalités de la variable dépendante CHAUD.

\section{Avec :}

CHAUD = 1 si l'entreprise a procédé au changement de son auditeur à la fin de son mandat, 0 sinon. Le tableau 5 affiche les résultats y afférents.

Tableau 5:-Tests pour échantillons indépendants

\begin{tabular}{|l|c|c|c|}
\hline & \multicolumn{3}{|c|}{ CHGAUD } \\
\hline Variables continues & 0 & 1 & z-stat \\
$(\mathrm{p})$ & $(\mathrm{N}=37)$ & $(\mathrm{N}=32)$ & \\
\hline
\end{tabular}




\begin{tabular}{|l|l|c|c|c|}
\hline$\Delta$ PRMANG & $\mathrm{M}$ & -0.558 & -0.002 & -0.573 \\
\hline & $\mathrm{m}$ & 0 & 0 & $(0.567)$ \\
\hline$\Delta$ PLACT & $\mathrm{M}$ & 2.96 & 3.044 & -0.346 \\
\hline & $\mathrm{m}$ & 0 & 0 & $(0.729)$ \\
\hline$\Delta$ LTDTA & $\mathrm{M}$ & 0.0305 & -0.0094 & $\mathbf{- 1 . 6 9 7}$ \\
\hline & $\mathrm{m}$ & 0.0044 & -0.0155 & $\mathbf{( 0 . 0 9 0 )}$ \\
\hline$\Delta$ TACTF & $\mathrm{M}$ & 0.172 & 0.053 & -1.273 \\
\hline & $\mathrm{m}$ & 0.125 & 0.039 & $(0.203)$ \\
\hline Variables modales & & 0 & 1 & $\mathrm{Khi} 2$ \\
& $\mathrm{MD}$ & $(\mathrm{N}=32)$ & $(\mathrm{N}=37)$ & $(\mathrm{p})$ \\
\hline$\Delta$ CHGDRG & 0 & 31 & 33 & $\mathbf{2 . 7 9 3}$ \\
\hline & 1 & 1 & 35 & $\mathbf{( 0 . 0 9 5})$ \\
\hline$\Delta$ RESV & 0 & 32 & 2 & $\mathbf{1 8 . 2 4 1}$ \\
\hline & 1 & 0 & $\mathbf{0 . 0 0 0})$ \\
\hline
\end{tabular}

Statistiques descriptives pour les variables explicatives continues: moyennes (M). médiane (m). Test non paramétrique pour deux échantillons indépendants de Mann-Whitney : Statistique z de test modalité 1 vs. Modalité 0 (probabilité critique bilatérale asymptotique). Les résultats significatifs $(\mathrm{p}<10 \%)$ sont repérés en gras. $\triangle \mathrm{PRMANG}=$ variation du pourcentage de capital détenu par le dirigeant entre $(\mathrm{t}-3)$ et $(\mathrm{t}) . \triangle \mathrm{PLACT}=$ variation du pourcentage de capital détenu par les trois principaux actionnaires entre (t-3) et (t). $\Delta$ LTDTA= variation du ratio d'endettement à terme entre $(\mathrm{t}-3)$ et $(\mathrm{t}) . \triangle \mathrm{TACTF}=$ pourcentage de variation du total actif de l'entreprise entre $(\mathrm{t}-3)$ et $(\mathrm{t})$.

Test d'indépendance du khi-deux à un degré de liberté pour les variables binaires. sont reportées : statistique du khi deux. probabilité critique bilatérale khi-deux $(\mathrm{p})$. Les résultats significatifs $(\mathrm{p}<10 \%)$ sont repérés en gras. $\triangle \mathrm{CHGDRG}=$ variable binaire codée 1 si l'entreprise a procédé à un changement du dirigeant durant la période $(\mathrm{t}-3)$ et $\mathrm{t} ; 0$ sinon. $\triangle \mathrm{RESV}=$ variable binaire codée 1 si le rapport d'audit de l'entreprise contient une réserve durant la période $(\mathrm{t}-3)$ et $(\mathrm{t})$.

Les statistiques du test de Mann-Whitney révèlent très peu de différences significatives pour les variables continues. En effet, nos résultats précédents s'avèrent robustes concernant l'effet de la propriété managériale et la concentration de propriété sur la décision du changement de l'auditeur. Toutefois, il s'avère que la variation comparée du ratio d'endettement à terme entre les entreprises qui décident de changer leurs auditeurs versus celles qui décident de renouveler le mandat de l'ancien auditeur crée la différence. En effet, la statistique du test de Mann Withney ressort significative au seuil de $10 \%$ pour l'endettement à terme ( $\triangle$ LTDTA). Nous concluons que la variation à la hausse de l'endettement à terme chez les entreprises tunisiennes non financières cotées engendre un renouvellement du mandat de l'ancien auditeur. Par ailleurs, les résultats du tableau 5 montrent que le changement du dirigeant influence significativement la décision du changement d'auditeur (au seuil de 1\%o) ce qui valide encore nos précédents résultats. Ceci confirme aussi l'idée selon laquelle le processus de désignation des auditeurs externes est largement dominé par les détenteurs du pouvoir exécutif qui supervisent et influencent également le processus de production de l'information comptable. Par ailleurs, l'insertion de réserves dans le rapport d'audit affecte significativement la décision de changer d'auditeur (au seuil de 1\%o). Ceci peut être interprété par le fait que les entreprises tunisiennes cotées tendent à changer leur auditeur en place à la fin de son mandat à la suite de la réception d'un rapport d'audit avec réserves.

\section{Conclusion:-}

Cette étude a pour objectif d'identifier les déterminants de la décision du changement de l'auditeur externe. Nous nous sommes basés essentiellement sur la théorie d'agence et l'impact des relations conflictuelles entre les différents intervenants liés à l'entreprise. Notre échantillon est composé par 23 entreprises tunisiennes non financières cotées et la période d'étude s'étale entre 2007 et 2017. Nous avons procédé à l'estimation logit de notre modèle d'étude. Nous avons conclu que le changement du dirigeant détermine le changement d'auditeur dans le contexte tunisien. Nous expliquons ceci par le fait qu'un nouveau dirigeant en place a besoin de rassurer les actionnaires et les investisseurs de sa nouvelle démarche dans l'entreprise. Ceci engendre un changement d'auditeur.

Dans le cadre des tests de robustesse, nous avons recouru au test non paramétrique de Mann-Withney. Nous avons extrait un échantillon de renouvellement du mandat de l'auditeur et un échantillon de changement d'auditeur sur la période 2009-2017. Nous avons montré que la variation de l'endettement à terme, le changement du dirigeant et l'émission de rapport avec réserves peuvent résulter à un changement d'auditeur. 
Nos résultats s'avèrent très intéressants à plusieurs égards. En effet, d'un point méthodologique, nous nous sommes basés sur une étude statique et dynamique pour identifier les déterminants du changement de l'auditeur externe dans le contexte tunisien. Sur le plan théorique, notre étude s'insère dans le cadre d'un contexte particulier par rapport aux études antérieures. En effet, alors que la majorité des études antérieures s'insèrent dans le contexte des pays développé, notre étude est réalisée dans un des pays émergents à culture particulière et se caractérisant par l'importance des relations personnelles dans le domaine professionnel. Sur le plan pratique, notre étude révèle que le dirigeant exécutif a une influence indirecte sur la décision du changement de l'auditeur. En effet, malgré que la loi réserve cette décision à l'assemblée générale des actionnaires, ceci n'exclut pas l'intervention du dirigeant dans ce processus. Un effort supplémentaire de la part du législateur tunisien est à fournir dans ce sens afin de préserver l'indépendance de l'auditeur.

Des recherches futures seraient intéressantes en vue d'élargir le champ de la notre et d'intégrer l'aspect de la direction du changement de l'auditeur et d'en identifier les déterminants. Elles pourraient aussi intégrer l'aspect de l'introduction en bourse qui constitue un évènement très important de la vie de l'entreprise et qui peut occasionner aussi un changement d'auditeur.

\section{Bibliographie:-}

1. Aguilar N.G., Barbadillo E.R. (2003) 'Do spanish firms change auditor to avoid a qualified audit report?', International Journal of Auditing, vol. 7(1), pp. 37-53.

2. Aroh J., Odum A., Odum C. (2017) 'Determinants of auditor switch: Evidence from quoted companies in Nigeria', International Journal of Management Research and Business Strategy, vol. 6(4), pp. 26-41.

3. Ball F., Tyler J., Wells P. (2015) 'Is audit quality impacted by auditor relationships?', Journal of Contemporary Accounting and Economics, vol. 11 (2), pp. 166-181.

4. Beattie V., Fearnley S. (1998) 'Audit market competition: auditor changes and the impact of tendering', The British Accounting Review, vol. 30(3), pp. 261-289.

5. Cameran M., Negri G., Pettinicchio A. (2015) 'The auditor mandatory rotation rule: the state of the art', The Journal of Financial Perspectives, vol. 3(2), pp.1-29.

6. Chadegani A., Mohamed Z., Jari A. (2011) 'The Determinant factors of auditor switch among companies listed on Tehran stock exchange', International Research Journal of Finance and Economics, vol. 80(80), pp. 158-168.

7. Chang W. C., Choy, H. L., Lin H. Y., Koo M. (2019) 'The determinants and effects of clients following audit partners who switch audit firms', European Accounting Review, In Press.

8. Chen K., Zhou J. (2007) 'Audit committee, board characteristics, and auditor switch decisions by Andersen's clients', Contemporary Accounting Research, vol. 24(4), pp.1085-1117.

9. Choy H., Lin H., Koo M. (2019) 'The determinants and effects of clients following audit partners who switch audit firms', European Accounting Review, In Press.

10. Corbella S., Florio C., Gotti G., Mastrolia S. (2015) 'Audit firm rotation, audit fees and audit quality: the experience of Italian public companies', Journal of International Accounting, Auditing and Taxation, vol. 25, pp. 46-66.

11. Darmayanti N. (2017) 'The effect of audit opinion, financial distress, client size, management turn and KAP size on auditor switching', Journal of Economics, Business and Accountancy Ventura, vol. 20(2), pp. 237-248.

12. DeFond M. (1992) 'The Association between changes in client firm agency costs and auditor switching', Auditing: A Journal of Practice and Theory, vol. 11(1), pp.16-31.

13. Faiza S. N., Nazri S. M., Smith M., Ismail Z. (2012) 'Factors influencing auditor change: evidence from Malaysia', Asian Review of Accounting, vol. 20(3), pp.222-240.

14. Francis J. R., Mehta M. N., Zhao W. (2017) 'Audit office reputation shocks from gains and losses of major industry clients', Contemporary Accounting Research, vol. 34(4), pp. 1922-1974.

15. Ghosh A., Lustgarten S. (2006) 'Pricing of Initial Audit Engagements by Large and Small Audit Firms', Contemporary Accounting Research, vol. 23(2), pp. 333-368.

16. Heliodoro P., Carreira F., Lopes M. (2016) 'The Change of auditor: The Portugese Case', Spanish Accounting Review, vol. 19(2), pp. 181-186.

17. Hudaib M., Cooke T. (2005) 'The impact of managing director changes and financial distress on audit qualification and auditor switching', Journal of Business Finance and Accounting, vol. 32(9-10), pp. 17031739.

18. Huson J., Shamsher M., Annuar M., Arief M. (2000) 'Auditor switch decision of malaysian listed firms: Tests of determinants and wealth effect', Pertanika Journal of Social Science and Humanities, vol. 8(2), pp. 77- 90. 
19. Jensen M., Meckling W. (1976) 'Theory of the firm: managerial behaviour, agency costs and ownership structure', Journal of Financial Economics, vol. 3(4), pp. 305-360.

20. Johnson W., Lys T. (1990) 'The market for audit services: Evidence from voluntary auditor changes', Journal of Accounting and Economics, vol. 12(1-3), pp. 281-308.

21. Kervin, J.B. (1992) Methods for business research, New York, Harpet Collins.

22. Khasharmeh H. (2015) 'Determinants of auditor switching in Bahraini's listed companies: an empirical study', European Journal of Accounting, Auditing and Finance Research, vol. 3(11), pp.73-99.

23. Kuo L. C., Lin C. J., Lin H. L. (2016) 'Auditor switch decisions under forced auditor change: evidence from China', Asian Review of Accounting, vol. 24(1), pp.69-89.

24. Liu M. (2007) 'Corporate governance, auditor choice and auditor switch: Evidence from China', Thèse de doctorat, Hong Kong Baptist University.

25. Patterson E.R., Smith J.R., Tiras S.L. (2019) 'The effects of auditor on fraud and its detection', The Accounting Review, In Press.

26. Piot C. (2003) 'Coûts d'agence et changements de commissaire aux comptes : une approche empirique', Comptabilité Contrôle Audit, vol.9(2), pp.5-30.

27. Rennie M., Kopp L., Lemon W. (2014) 'Auditor-client disagreements and independence: an exploratory field study', Research on Professional Responsibility and Ethics in Accounting, vol.18, pp.131-166.

28. Schwartz K.B., Menon K. (1985) 'Auditor switches by failing firms', The Accounting Review, vol. 60(2), pp. 248-261.

29. Shahnaz I., Nurasyikin J., Wan Zanani W. (2008) 'The impact of board composition, ownership and CEO duality on audit quality: The Malaysian evidence', Management and Accounting Review, vol. 7(2), pp. 17-28.

30. Tu G. (2012) 'Controller changes and auditor changes', China Journal of Accounting Research, vol. 5(1), pp. 45-58.

31. Zhang S., Ye K., Cui Y., Zang W. (2019) 'Large shareholder incentives and auditor choice', Auditing: A Journal of Practice and Theory, In Press. 